\title{
Serine proteinase inhibitors from nematodes and the arms race between host and pathogen
}

\author{
Xingxing Zang and Rick M. Maizels
}

\begin{abstract}
Serine proteinase inhibitors are encoded by a large gene family of long evolutionary standing. Recent discoveries of parasite proteins that inhibit human serine proteinases, together with the complete genomic sequence from Caenorhabditis elegans, have provided a set of new serine proteinase inhibitors from more primitive metazoan animals such as nematodes. The structural features (e.g. reactive centre residues), gene organization (including intron arrangements) and inhibitory function and targets (e.g. inflammatory and coagulation pathway proteinase) all contribute important new insights into proteinase inhibitor evolution. Some parasite products have evolved that block enzymes in the mammalian host, but the human host responds with a significant immune response to the parasite inhibitors. Thus, infection produces a finely balanced conflict between host and pathogen at the molecular level, and this might have accelerated the evolution of these proteins in parasitic species as well as their hosts.
\end{abstract}

\footnotetext{
Xingxing Zang

Howard Hughes Medical

Institute, Dept of

Molecular and Cell

Biology, University of

California, Berkeley,

CA 94720-3200, USA.

e-mail:xxzang@

uclink4.berkeley.edu

Rick M. Maizels*

Institute of Cell, Animal

and Population Biology,

University of Edinburgh,

West Mains Road,

Edinburgh, UK EH9 3J T.

*e-mail:

r.maizels@ed.ac.uk
}

Serine proteinases are ubiquitous enzymes involved in many physiologicial events in all taxonomic groups.

Their activity, if uncontrolled, can be destructive to the cell or organism and must therefore be precisely regulated by endogenous inhibitors. Serine proteinase inhibitors are categorized according to primary sequence, structural motifs and mechanism of binding. Among these protein families, the serpins are of particular interest ${ }^{1}$. Serpins are large proteins, more than 100 of which have been identified in species as diverse as vertebrates, insects, plants and viruses ${ }^{2}$. In mammals, they are key regulatory proteins involved in essential extracellular functions such as complement activation, fibrinolysis, coagulation and inflammation ${ }^{1}$. In addition, some serpins have unique intracellular roles in the control of cell mobility ${ }^{3}$, chromatin folding ${ }^{4}$ and Toll signalling 5 . Their importance is attested to by the 90 or more natural serpin mutations now associated with human diseases such as thrombosis and emphysema ${ }^{6}$.

Nematodes occupy a lowly place on the invertebrate evolutionary tree ${ }^{7}$, but are of prominent interest for two reasons. First, the genome sequence of the free-living nematode Caenorhabditis elegans has afforded the first view of a metazoan gene complement ${ }^{8}$; and second, nematode worm parasites are among the most prevalent in tropical countries with more than one billion infected people in the world today. One of the challenges for modern molecular medicine will be to exploit the information from C. elegans to solve major health problems of populations afflicted by parasitic diseases such as ascariasis and filariasis.

Parasite nematode genomics is a relatively new field ${ }^{9}$, but already two of the most interesting gene families to be found encode serine proteinase inhibitors. This article describes a family of nematode proteinase inhibitors with homology to mammalian serpins, as well as a distinct set of lower-molecularweight inhibitors first discovered by biochemical analysis of the human roundworm Ascaris ${ }^{10}$. Taking these two examples into account, it thus appears that parasitic nematodes have evolved two parallel strategies for interfering with mammalian serine protease-dependent processes.

\section{Nematode serpins: genes and proteins}

Table 1 is a compilation of all likely nematode serine proteinase inhibitor genes available from accessible databases. The first part of the table presents members of the serpin family, which currently encompasses eight C. elegans genes ${ }^{11}$ and eight genes from five parasitic species such as Ascaris suum and the filarial nematodes Brugia malayi and Onchocerca volvulus, the causative agents of lymphatic filariasis and river blindness, respectively. For comparison, Table 1 also lists serpins from Schistosoma, a genus of trematode flukes responsible for schistosomiasis in 300 million patients. The second section of Table 1 describes the distinct family of small serine protease inhibitors, of which 14 are known in nematode parasitic species (see below).

The serpin family has been constructed on the basis of sequence similarities at the protein level ${ }^{2}$. All members of the serpin family are thought to share a common highly ordered tertiary structure, which consists of nine $\alpha$ helices and three $\beta$ sheets ${ }^{24}$. The functional domain is near the C-terminus, a small region termed the reactive-site loop exposed at the surface of the protein (Fig. 1). This loop contains a scissile bond, a cleavable bait that traps the protease. The reaction with target enzymes is dramatic: the protease cleaves the serpin loop but remains attached through its active-site serine, and the entire reactive centre springs across the serpin molecule, a distance of $71 \AA$ (Ref. 25). In this process, the serpin loop disrupts the structure of the target protease, rendering it liable to degradation. 
Table 1 Comparison of the properties and features of serine proteinase inhibitors in nematodes and other helminthsa.

\begin{tabular}{|c|c|c|}
\hline Protein name & $\begin{array}{l}\text { Target or closest } \\
\text { mammalian homologue }\end{array}$ & Refs or GenBank accession numberb \\
\hline \multicolumn{3}{|c|}{ Nematode serpins } \\
\hline \multicolumn{3}{|l|}{ Ascaris suum } \\
\hline As-SPN & Human antithrombin III & AW165870 \\
\hline \multicolumn{3}{|l|}{ Brugia malayi } \\
\hline$B m-S P N-1$ & Mouse Spi3 & 12 \\
\hline$B m-S P N-2$ & Cathepsin G, neutrophil elastase & 13 \\
\hline$B m-S P N-3$ & Mouse SCCA2 & $\begin{array}{l}\text { AA990949, Al133763, } \\
\text { H48987 }\end{array}$ \\
\hline \multicolumn{3}{|c|}{ Caenorhabditis elegans } \\
\hline Ce-SPN-1 & Mouse SCCA2 & U50301 \\
\hline Ce-SPN-2 & Human SCCA2 & U50301 \\
\hline CeSPN-3 & Human pancpin & U97008 \\
\hline Ce-SPN-4 & Human SCCA2 & U97008 \\
\hline CeSPN-5 & Mouse SCCA2 & AF026209 \\
\hline CeSPN-6 & Human neuroserpin & AF026209 \\
\hline Ce-SPN-7 & Mouse SCCA 2 & Z81496 \\
\hline Ce-SPN-8 & Human protease inhibitor 8 & Z81496 \\
\hline \multicolumn{3}{|c|}{ Onchocerca volvulus } \\
\hline Ov-SPN-1 & Mouse neuroserpin & 14 \\
\hline$O v-S P N-2$ & Porcine elastase inhibitor & AA625018 \\
\hline \multicolumn{3}{|c|}{ Onchocerca ochengi } \\
\hline Oo-SPN & Mouse neuroserpin & Al363545 \\
\hline \multicolumn{3}{|c|}{ Trichostrongylus vitrinus } \\
\hline$T V-S P N$ & Mouse Spi3 & $\begin{array}{l}\text { TVY12230, TVY12231, } \\
\text { TVY12232, TVY } 12233\end{array}$ \\
\hline \multicolumn{3}{|c|}{ Schistosoma mansoni (Trematode) } \\
\hline Smpi56 & Elastase & 15 \\
\hline \multicolumn{3}{|c|}{ Schistosoma haematobium (Trematode) } \\
\hline SHSP1 & Human SCCA2 & 16 \\
\hline \multicolumn{3}{|c|}{ Nematodesmapins } \\
\hline \multicolumn{3}{|c|}{ Anisakis simplex } \\
\hline ASPI-1 & Elastase & 17,18 \\
\hline ASPI-2 & Elastase & 17,18 \\
\hline ASPI-3 & & 17,18 \\
\hline \multicolumn{3}{|l|}{ Ascaris suum } \\
\hline ICE1 & Chymotrypsin, elastase & 19 \\
\hline ICE2-5 & Chymotrypsin, elastase & 19 \\
\hline ITR1 & Trypsin & 20 \\
\hline ITR2 & Trypsin & 20 \\
\hline \multicolumn{3}{|c|}{ Ancylostoma caninum } \\
\hline AcAP5 & Factor Xa, Xla & 21 \\
\hline AcAP6 & Factor Xa & 21 \\
\hline AcAPC2 & Factor VIla/TF & 21 \\
\hline \multicolumn{3}{|c|}{ Onchocerca volvulus } \\
\hline Ov-SPI-1 & & 14 \\
\hline Ov-SPI-2 & & $\begin{array}{l}\text { AA618955, OVAA94747, } \\
\text { AA670498, OVAA94039 }\end{array}$ \\
\hline \multicolumn{3}{|l|}{ Trichuris suis } \\
\hline Ts-TCl & Trypsin, chymotrypsin & 22 \\
\hline Ts-CEI & $\begin{array}{l}\text { Chymotrypsin, elastase, } \\
\text { chymase, cathepsin G }\end{array}$ & 23 \\
\hline
\end{tabular}

Although nematode serpins have low overall homology to the serpins from mammalian species, their sequences are identical or conserved at most of the key amino acid positions. For example, the 'serpin signature', which includes the hinge distal to the reactive loop, is well conserved (Fig. 1). However, analysis of the 'serpin motif' (in the proximal hinge) is ambiguous. Here, there are only two residues in Bm-SPN-2 (E353 and G355) that are also found in all other functional serpins ${ }^{26}$. Few of the other residues in $\mathrm{Bm}$-SPN-2 follow the consensus for small nonpolar side-chains, which would be most compatible with the 


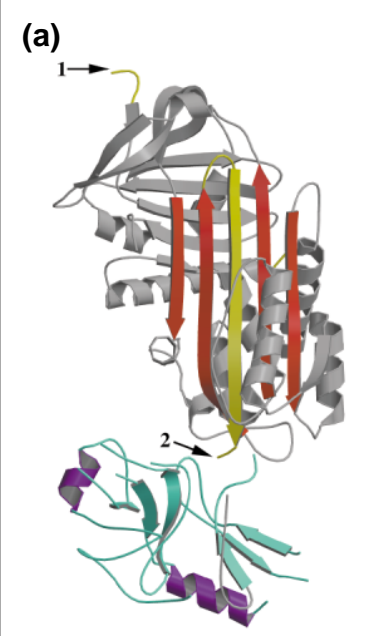

(b)

$P_{1} r_{P 1}$

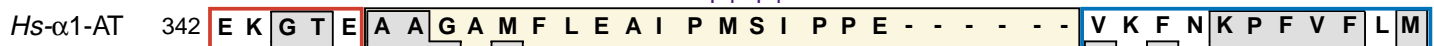

Bm-SPN-1 343 E K G T E S A A A T T I I E L E D R M G S S R N - . . - F F A A D Q P F L F A I

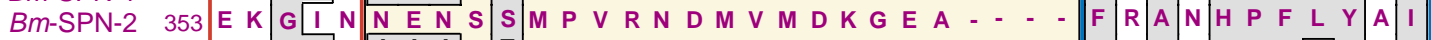

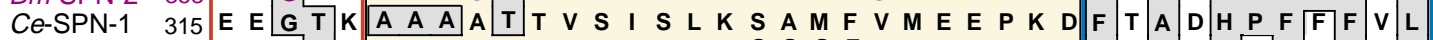
Ce-SPN-2 315 - - - T T K

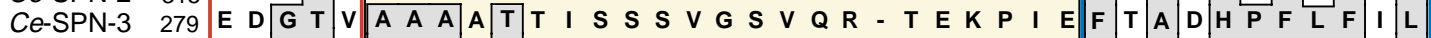
Ce-SPN-4 315 E D

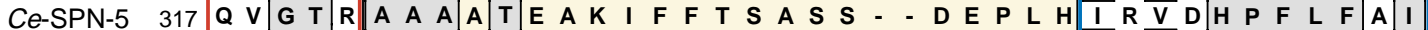
Ce-SPN-6 310 E D G T T A A A A S A F K V Q L E M M I M - - A E P T Q Ce-SPN-7 310 K M G T T A A A V Ce-SPN-8 295 K M $\mathbf{G}$ T T SHSP1 350 E S

Serpin motif

Reactive-site loop

Serpin signature

Fig. 1 Structure and sequences of serpins. (a) Structure of a serpin ( $\alpha 1$-antitrypsin, top)-protease (trypsin, bottom) complex. The reactive-site loop of serpin is shown as a yellow strand; five $\beta$ strands are shown as red arrows. Before interaction with target protease, the side-chain of Met358 is at position 1 and is adjacent to the scissile bond. Following cleavage by protease, the larger part of the reactive loop moves to align with the $\beta$ strands, and Met358 comes to rest at position 2 . The structure is adapted from Protein Data Bank (PDB) under accession number 1EZX. (b) Amino acid sequences of nematode serpins in the region of the reactive-site loop, and comparison with human $\alpha 1$-antitrypsin (Hs- $\alpha 1-A T)$. The serpin motif is boxed in red, the reactive-site loop shaded in yellow, and the serpin signature framed in blue. The alignment of sequences was made with MacVector (version 6.5) and manually adjusted to give the best fit. Gaps were introduced into sequences for optimal alignment. The two Brugia malayi serpins are shown in purple lettering. Residues that are identical or similar in eight or more of the ten nematode serpins are shaded in grey. The scissile bond is marked with a red arrow.
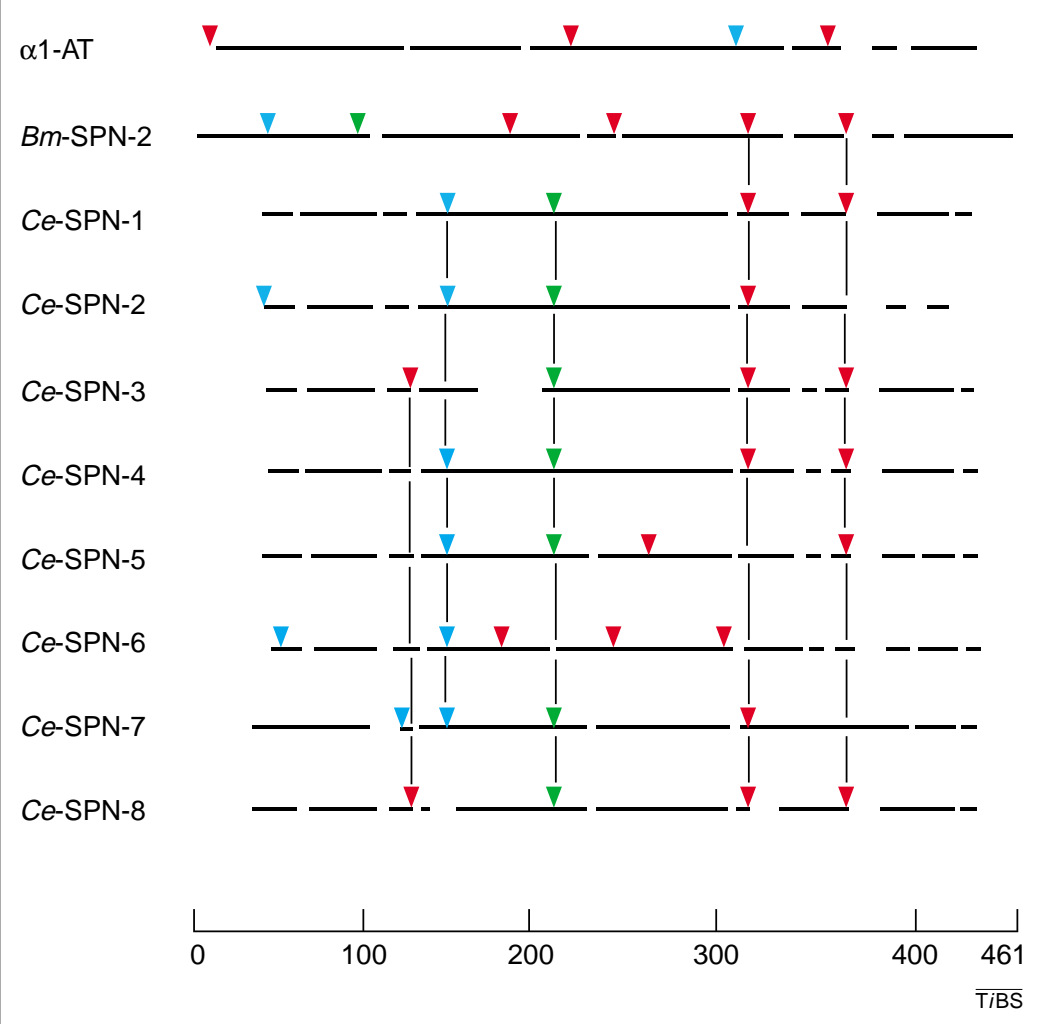

Fig. 2. Intron and exon positions in nematode serpin genes. Horizontal lines indicate coding regions, with gaps introduced to optimize alignment. Arrows indicate positions of introns. Sequences were aligned on the basis of amino acid similarity; the scale shows amino acid positions beginning with the first methionine of $B m$-SPN-2. Introns are shaded according to their phase : 0 (red, intron insertion precisely between two triplet codons); 1 (green, intron insertion after the first base of a codon); or 2 (blue, intron insertion after the second base). Vertical lines connect introns considered to be in identical positions. transformation associated with protease inactivation $^{6,26}$. Thus, the consensus sequence might require re-definition in the light of these new, phylogenetically distant genes from nematodes and other invertebrates.

The amino acid sequence of the reactive-site loop itself, however, is hypervariable among both mammalian and nematode serpins (Fig. 1). This hypervariability appears to be a consequence of unusually high rates of nonsynonymous substitution within the reactive-site loops ${ }^{27,28}$. Such alterations, particularly at the $\mathrm{P} 1$ position immediately proximal to the scissile bond of the reactive centre, can cause dramatic changes in the proteinase selectivity of a serpin. The accumulation of amino acid substitutions in the catalytic or functional domains of proteins is relatively rare, with the important exception of immune-system receptors selected to respond to an infinite number of antigens. The rapid diversification of the reactive centre in serpins might therefore have facilitated the emergence of novel adaptive properties, thereby allowing a nematode serpin to target a mammalian host enzyme ${ }^{13}$ - a key step in the 'evolutionary arms race'.

\section{Gene structure and evolution of serpins}

At the level of gene structure, comparisons of the eight C. elegans serpin genes and the one B. malayi gene for which complete genomic sequences are available, show many interesting characteristics (Fig. 2). Introns map in a varied pattern, with not a single position conserved even within C. elegans, although two at the 3 ' end of the gene occur within both nematode species. As B. malayi and C. elegans diverged many hundred million years ago, this conservation implies a functional constraint for the C-terminus of the protein, which perhaps corresponds to the reactive-site loop. Interestingly, two of the four C. elegans serpin genes in which one of the two conserved introns is missing (Ce-SPN-2 and Ce-SPN-5) are highly divergent in this loop (Fig. 1).

The original studies on vertebrate serpin genes ${ }^{29}$ favoured an 'introns-early' hypothesis ${ }^{30}$, which 


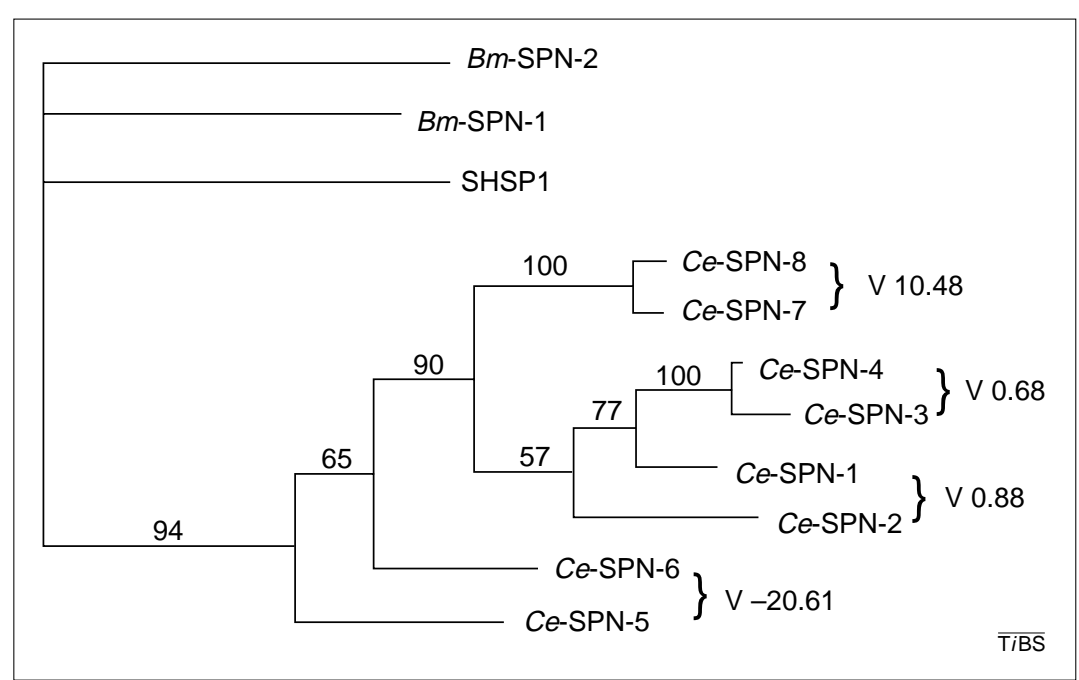

Fig. 3. Phylogeny of nematode serpins for which full sequences are available. The nematode serpins are grouped into two major clusters: all Caenorhabditis elegans serpins are grouped together whereas the other serpins are divergent. Positions of $C$. elegans serpin genes on chromosome $V$ are indicated. The phylogenetic tree was generated by PAUP (4.0.3) using sequence alignment by removal of significant inserts and trimming the $\mathrm{C}$ - and $\mathrm{N}$-terminal extensions. The percentage bootstrap support for each relationship is denoted above the branches for each clade.

suggested that numerous introns used to assemble the first genes were simply lost during evolution, rather than there being a more dynamic 'gain-andloss' model ${ }^{31}$. However, the comparison presented in Fig. 3 would require 12 ancestral introns simply to account for serpin genes in two nematode species, plus four more to encompass the vertebrate descendants. If additional species prove as diverse as B. malayi and C. elegans with respect to serpin gene structure, then intron gain-and-loss would be a more likely model of serpin gene evolution, as postulated for other gene families ${ }^{31}$.

All eight C. elegans serpin genes are clustered in three regions of chromosome V. An interesting relationship between chromosomal location and serpin protein sequence identity can be discerned, providing a glimpse into their evolutionary history. The amino acid sequences of the serpins mapping to V0.68 ( $\mathrm{Ce}$-SPN-3 and $\mathrm{Ce}$-SPN-4) and V10.48 (Ce-SPN-7 and $C e-\mathrm{SPN}-8)$ show the highest level of identity ( $87 \%$ and $78 \%$, respectively). Phylogenetic analysis groups these two pairs closely together, which indicates that a local duplication event occurred (Fig. 3). The other serpins are slightly more diverse, with $C e-\mathrm{SPN}-1$ being closer to $C e-\mathrm{SPN}-4$ than to its neighbour $\mathrm{Ce}$-SPN-2 on V0.88. The clustering of C. elegans serpins might provide new opportunities to study coordinate gene regulation and the evolution of gene families. Is the genomic aggregation of the serpin genes simply a consequence of using gene amplification and divergent evolution to increase the repertoire of proteinase inhibitors? This might be the case, as $C e$-SPN-7 and $C e$-SPN-8 have very different reactive-site loops (Fig. 1). Alternatively, there could be selective pressure to retain the genomic organization of the serpin cluster, so that gene expression can be coordinately or temporally regulated in response to different environmental or developmental cues, respectively. It is hoped that, as functional analysis of C. elegans genes proceeds, the relationship between gene organization and position, and serpin specificity and expression, will provide an alternate way to advance the fundamental knowledge of evolutionary relatedness.

\section{Smapins: small serine proteinase inhibitors}

One of the first polypeptides sequenced from a parasitic nematode is now recognized as the prototypic member of a new family of serine proteinase inhibitors, all of which comprise less than 100 amino acid residues. Fourteen members of this family have been reported, or they can be found by homology searching in accessible databases (Table 1) on parasitic nematodes, but there are no other recognizable relatives from nematodes or any other organism. Because the small serine proteinase inhibitors show no structural similarity to other better-known serine proteinase inhibitor families, the authors suggest that a new name is assigned to them - the smapins.

The most striking characteristic of this smapin family is the universal presence of ten cysteine residues that form five disulphide bonds. Structural studies have determined the location of these bridges, two of which are positioned either side of the reactivesite loop (Fig. 4). Smapin structures from both A. suum (ITR1) ${ }^{20}$ and Ancylostoma caninum (AcAPc2) ${ }^{32}$, determined by nuclear magnetic resonance (NMR), contain two $\beta$ sheets, each composed of two antiparallel strands, with the remainder consisting of turns and extended loops. The protein cores are occupied by the disulphide bridges which, together with an elaborate network of electrostatic and hydrogen-bonding interaction, might provide the structural basis for the protease and heat resistance of these inhibitors. NMR also showed an interesting $\mathrm{pH}$ dependent conformational transition of the reactivesite loop ${ }^{20}$, which might be related to the parasite's requirement to traverse hostile environments that have varying $\mathrm{pH}$ conditions, such as the gut.

The crystal structure of smapin ICE (inhibitor of chymotrypsin/elastase)-1 from $A$. suum bound to porcine elastase reveals a remarkable interpenetration between proteinase and the inhibitor $^{19}$. Not only does the $\mathrm{P} 1$ residue of the inhibitor penetrate into the enzyme's primary specificity pocket, but the elastase reciprocates by projecting an arginine residue through a pore adjacent to the $\mathrm{P} 1$ residue of the inhibitor. Like serpins, reactive-site loops of the smapin family are hypervariable, and selective pressure by host enzymes might have contributed to the hypervariability ${ }^{17,33}$.

These structural studies confirm the proposition that the smapin family radically differs from known inhibitor families in tertiary structure and topography of disulphide bridges, and thus represents a novel family of inhibitors. 


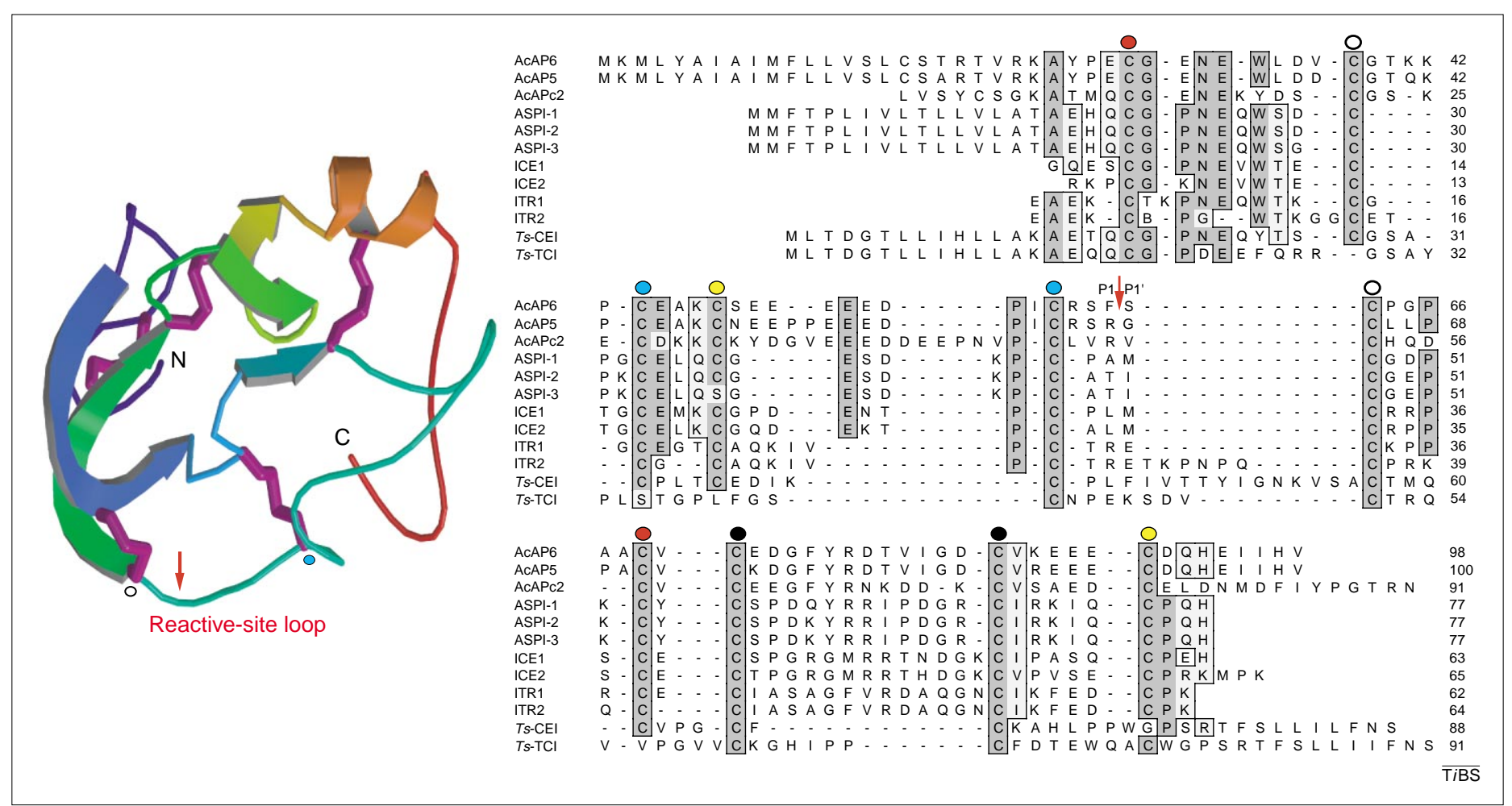

Fig. 4. The small serine proteinase inhibitor (smapin) family from nematodes. The smapins contain ten conserved cysteines, which are shown in the AcAPc2 structure adapted from the Protein Data Bank (PDB) (accession number 1COU) (left), and in the sequence alignment (right). The disulphide pairing is shown by coloured ellipses above the sequences and the scissile bond is marked with a red arrow. Grey shaded areas represent identical or similar residues. In the structural presentation, the reactive-site loop is shown, with the scissile bond marked with a red arrow and the adjacent cysteine residues coloured according to the scheme used in the alignment. Note that ACAPC2, ICE1, ICE2, ITR1 and ITR2 are sequences derived from mature polypeptides, with no signal sequence present. Abbreviations: ACAP, Ancylostoma caninum anticoagulant protein; ICE, inhibitor of chymotrypsin/elastase; ITR, inhibitor of trypsin.

\section{Serpin and smapin functions}

In view of the key role that mammalian serpins play in the regulation of biological processes, one can imagine that pathogens might themselves encode serpins and use them to block host defence functions. Serpins from viruses have been implicated in pathogen evasion of the host immune system. The only nematode serpin for which experimental evidence indicates an evasive function is $\mathrm{Bm}-\mathrm{SPN}-2$. $B m$-SPN-2 specifically inhibits two human neutrophil-derived serine proteinases, cathepsin $\mathrm{G}$ and elastase ${ }^{13}$. It is interesting that $\mathrm{Bm}$-SPN-2 is expressed and secreted at a single point in the parasite life cycle by the blood-borne microfilariae ${ }^{13,34}$. Given that neutrophils are the predominant leukocyte in human blood, the release of $B m$-SPN-2 could be an appropriate strategy for the blood-living microfilarial stage. The consequences of $B m$-SPN-2 inhibition in vivo have yet to be studied, but neutrophil-derived cathepsin $\mathrm{G}$ is known to be an important chemokinetic stimulator of $\mathrm{T}$ lymphocytes and a chemoattractant for monocytes ${ }^{35}$, as well as a mediator of chemokine processing ${ }^{36}$. Less is known of $B m$-SPN-1, which is present at all stages of the parasite life cycle ${ }^{12}$. As it is expressed most highly in the mosquito-borne infective stage, where it is found as a complex, this serpin could exist to inhibit a cognate proteinase endogenous to the parasite ${ }^{12}$.

The functions of Schistosoma serpins are at present open to speculation ${ }^{37}$. These serpins are found either on or within the worm surface tegument and are present in higher-molecular-weight forms, indicating complex formation perhaps with a cognate proteinase $^{15,16}$. Smpi56 inhibits both Schistosoma and neutrophil elastases ${ }^{15}$. Consequently, its role could include both the physiological control of elastase within the schistosomes, and protection of the parasite from activated neutrophils during inflammation.

Although C. elegans has eight serpins, their physiological roles have yet to be established. The presumption will be that their primary role is the regulation of endogenous serine proteinases, given that none possess a signal peptide. However, it is conceivable that one or more has evolved to inhibit proteinases produced by pathogenic microorganisms, as postulated for families of serpins in mice ${ }^{28}$.

The role of smapin molecules has, however, been clarified in two distinct parasite systems. In Ascaris, a parasitic nematode that resides within the human and pig intestinal tract, smapins protect the worm from proteolytic degradation by the host's digestive enzymes $^{38}$. These inhibitors are located at the surface of the developing eggs, larvae and the epithelial surface of the worm's own gut, combining with host proteinases to form inactive enzyme-inhibitor complexes. It is possible that this mechanism not only protects Ascaris within a degradative environment but could also mask the surface of developing larvae, permitting them to evade the host's immune system as they migrate from the intestines to the liver and lungs. 


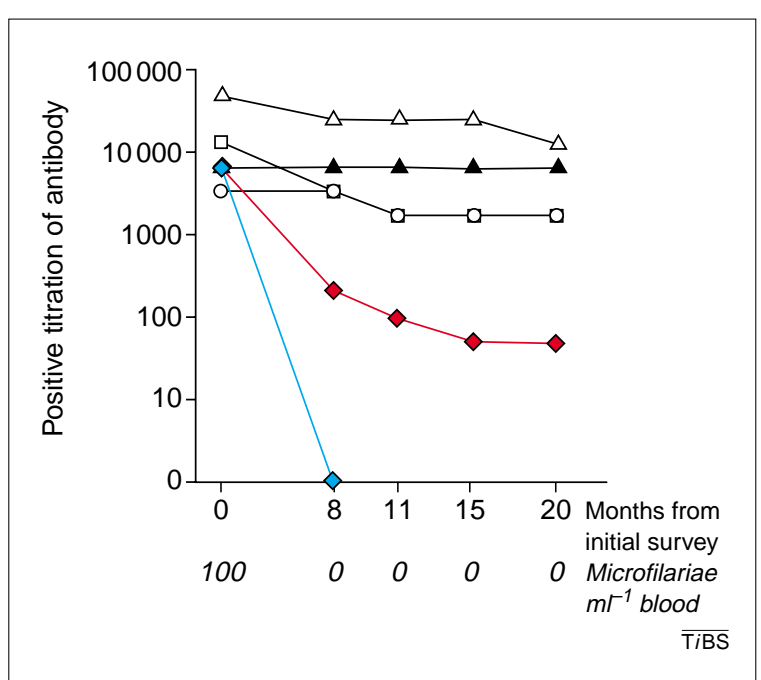

Fig. 5. $B m$-SPN-2-specific antibody lgG4 is a good diagnostic indicator of Brugia malayi infection. Kinetics of serum IgGl and IgG4 responses against $B m-S P N-2$, microfilarial antigens and adult antigens in a microfilaraemic patient before and after natural parasite clearance. The microfilarial and adult antigens are complex mixtures obtained by homogenization of these stages of parasite in phosphate buffer, and recovering the soluble fractions. Antibody levels were measured at the intervals indicated by enzyme-linked immunosorbent assay, using plates coated with the respective antigens at equivalent concentrations. Key: red diamonds, IgG1 to Bm-SPN-2; blue diamonds, IgG4 to Bm-SPN-2; circles, IgG1 to microfilariae antigens; squares, IgG4 to microfilariae antigens; closed triangles, IgG1 to adult antigens; open triangles, lgG4 to adult antigens.

In hookworms, the smapins are responsible for the long-known anticoagulant properties of these bloodfeeding parasites. Recently, three smapins have been identified in the $\operatorname{dog}$ hookworm $A$. caninum $^{39}$. Each inhibitor (AcAPc2, AcAP5 and AcAP6) specifically inhibits a different range of blood coagulation serine proteinase ${ }^{21}$ with greater potency than the lowmolecular-weight anticoagulant heparin ${ }^{40}$. AcAP6 inhibits factor Xa (fXa), whereas AcAP5 inhibits both fXa and fXIa. Both AcAP5 and AcAP6 inhibit thrombin formation by binding directly to the catalytic site of fXa.AcAPc2 is the most potent anticoagulant of all hookworm products, but shows little inhibition of purified $\mathrm{fXa}$ in solution. In fact, AcAPc2 does bind to $\mathrm{fXa}$, but at an exo-site distant from the active centre and, once bound to $\mathrm{Xa}, \mathrm{AcAPc} 2$ inhibits the fVIIa-tissue factor (VIIa-TF) complex, which is a central component of both intrinsic and extrinsic coagulation pathways ${ }^{21}$. AcAPc2 is subtly different from the mammalian anticoagulant tissue factor pathway inhibitor, which also shows fXa-dependent inhibition of VIIa-TF, and which is known to interact with the active site of each serine proteinase. From these studies, it appears that $A$. caninum has evolved a highly effective strategy for manipulation of the mammalian blood coagulation pathway with a novel mechanism distinct from those used by other haematophagous parasites or the mammalian host.

\section{Antigenicity of serpins to the host}

Pathogen survival strategies generally require the production of specific mediators, such as serpins, which interfere with host-protective mechanisms. However, by releasing such mediators, the invading organism might also be offering a target for the host immune system to recognize and attack. Recent data have suggested that serpins carry precisely this dual characteristic, and that infected hosts strongly recognize the antigenic differences between endogenous and parasite homologues.

$\mathrm{Bm}$-SPN-2 was first identified as an antigen that could elicit strong T-cell and antibody responses in human patients and laboratory model systems ${ }^{34}$. Patients with blood microfilariae display remarkably strong antibody responses to $\mathrm{Bm}$-SPN-2. The level of these antibodies declines sharply when parasites are cleared by drugs or the immune system (Fig. 5), although antibodies to other parasite antigens are still present. Therefore, the presence of $\mathrm{Bm}$-SPN-2-specific antibody is a useful molecular marker for infection so that, as an antigen, $\mathrm{Bm}$-SPN-2 could be advantageously used in a new diagnostic test for individual infection and community parasite load. The predominant isotypes of $\mathrm{Bm}$-SPN-2-specific antibody are IgG1 and IgG4 (Ref. 34), the latter being unusually well-represented in the serum antibody response to this antigen. This mirrors a well-established, but unexplained, link between microfilaraemic infection and high IgG4 antibody levels. Recent work has shown that human $\alpha 1$-antitrypsin, a physiological serpin that neutralizes neutrophil elastase, selectively upregulates B-cell differentiation towards IgE- and IgG4-secretion ${ }^{41}$. This effect was not observed with other mammalian serpins. The possibility that $B m$-SPN-2, which also neutralizes human neutrophil elastase $^{13}$, could be a key co-stimulus for IgG4 switching remaining open.

Relatively little is known of the antigenicity of the other nematode serpins and smapins, but this is clearly an area worthy of investigation. $B m-\mathrm{SPN}-1$ is recognized by antibodies from $B$. malayi-infected animals ${ }^{12}$, but its antigenicity in human infection has yet to be documented.

Three related species of schistosome parasites, Shistosoma haematobium, S. mansoni and S. japonicum, cause human schistosomiasis. Although there is extensive immunological cross-reactivity between all three species, the host's protective immunity is largely directed towards species-specific components. Therefore, antigens unique to one species might play a role in the development of resistance to infection. One such antigen is $S$. haematobium serpin SHSP1 (Ref. 42). SHSP1 induces a largely speciesspecific IgG4 and IgE response, whereas antibody from the same patients to total adult worm or egg antigens does not discriminate between species. After patients underwent chemotherapy, SHSP1-specific IgG4 levels dropped whereas IgE rose 50-100-fold, disproving the proposition that production of IgG4 and $\mathrm{IgE}$ is regulated in parallel. These data suggest that the mechanisms for generating IgE and IgG4 production in response to SHSP1 might differ. 
These studies raise several interesting questions. For example, has a molecule important to parasite immune evasion become the target of the host immune system? A parallel can be drawn with the HIVgp120 glycoprotein and the Plasmodium falciparum var antigen. If so, this would be an ironic but fitting twist to the continuous interaction between the host and the pathogen, termed the evolutionary arms race.

\section{Concluding remarks}

The presence of serine proteinase inhibitors in nematodes demonstrates that this important regulatory family appeared early in metazoan evolution. Parasite serine proteinase inhibitors are among the best examples of genes interactive with host physiological processes, whereas the newly completed genome sequence of C. elegans, coupled with the 'toolbox' of molecular techniques in this model system, promise a rapid and effective study of serpin gene functions. Parasite serpins can also serve as targets for host immune responses, thereby providing possibilities for novel vaccine development. Furthermore, the relatively primitive parasitic nematodes have co-evolved highly specific small serine proteinase inhibitors to interfere with host effector mechanisms and ensure survival. Recent work on Drosophila melanogaster serpins ${ }^{5}$ raises an interesting possibility that pathogens could produce serpins to inhibit Toll-mediated defence, one of the key pathways in innate immunity. Studying these inhibitors could provide new weapons for combating uncontrolled proteinase activity in human diseases. Indeed, AcAP5 and AcAPc2 are now undergoing clinical trials for use in humans as novel anticoagulants. In the same manner, the array of nematode serpins might, in time, provide a wealth of material for future study and therapeutic benefit.
Acknowledgements
We thank D.B. Guiliano and J . Parkinson for their invaluable help. serine protease inhibitor. Exp. Parasitol. 78, 121-131

1 Potempa, J.et al. (1994) The serpin family of proteinase inhibitors: structure, function, and regulation. J. Biol. Chem. 269, 15957-15600

2 Marshall, C.J. (1993) Evolutionary relationships among the serpins. Philos. Trans. R. Soc. London Ser B 342, 101-119

3 Stefansson, S. and Lawrence, D.A. (1996) The serpin PAI-1 inhibits cell migration by blocking integrin $\alpha_{\mathrm{V}} \beta_{3}$ binding to vitronectin. Nature 383, 441-443

4 Grigoryev, S.A. et al. (1999) MENT, a heterochromatin protein that mediates higher order chromatin folding, is a new serpin family member. J. Biol. Chem. 274, 5626-5636

5 Levashina, E.A. et al. (1999) Constitutive activation of toll-mediated antifungal defense in serpindeficient Drosophila. Science 285, 1917-1919

6 Stein, P.E. and Carrell, R.W. (1995) What do dysfunctional serpins tell us about molecular mobility and disease? Nat. Struct. Biol. 2, 96-113

7 Aguinaldo, A.M.A. et al. (1997) Evidence for a clade of nematodes, arthropods and other moulting animals. Nature 387, 489-493

8 The C.elegans Genome Consortium (1998) Genome sequence of Caenorhabditis elegans: a platform for investigating biology. Science 282, 2012-2018

9 Blaxter, M.L. et al. (1999) Parasitic helminth genomics. Parasitology 118, S39-S51

10 Grütter, M.G. (1994) Proteinase inhibitors: a new fold. Structure 2, 575-576

11 Whisstock, J.C.et al. (1999) Serpins in the Caenorhabditis elegans genome. Proteins $36,31-41$

12 Yenbutr, P. and Scott, A.L. (1995) Molecular cloning of a serine proteinase inhibitor from Brugia malayi. Infect. Immun. 63, 1745-1753

13 Zang, X. et al. (1999)A novel serpin expressed by the blood-borne microfilariae of the parasitic nematode Brugia malayi inhibits human neutrophil serine proteinases. Blood 94, 1418-1428

14 Lizotte-Waniewski, M. et al. (2000) Identification of potential vaccine and drug target candidates by expressed sequence tag analysis and immunoscreening of Onchocerca volvulus larval cDNA libraries. Infect. Immun. 68, 3491-3501

15 Ghendler, Y.et al. (1994) Schistosoma mansoni: isolation and characterization of Smpi56, a novel
16 Blanton, R.E.et al. (1994) Characterization of a native and recombinant Schistosoma haematobium serine protease inhibitor gene product. Mol. Biochem. Parasitol. 63, 1-11

17 Lu, C.C.et al. (1998) Anisakis simplex: mutational bursts in the reactive site centers of serine protease inhibitors from an ascarid nematode. Exp. Parasitol. 89, 257-261

18 Nguyen, T.T.et al. (1999) Expression and characterization of elastase inhibitors from the ascarid nematodes Anisakis simplex and Ascaris suum. Mol. Biochem. Parasitol. 102, 79-89

19 Huang, K. et al. (1994) The molecular structure of the complex of Ascaris chymotrypsin/elastase inhibitor with porcine elastase. Structure 2, 679-689

20 Grasberger, B.L. et al. (1994) High-resolution structure of Ascaris trypsin inhibitor in solution: direct evidence for a $\mathrm{pH}$-induced conformational transition in the reactive site. Structure 2, 669-678

21 Stanssens, P.et al. (1996) Anticoagulant repertoire of the hookworm Ancylostoma caninum. Proc. Natl. Acad. Sci. U. S. A. 93, 2149-2154

22 Rhoads, M.L.et al. (2000) Trichuris suis: a secretory serine protease inhibitor. Exp. Parasitol. 94, 1-7

23 Rhoads, M.L. et al. (2000) Trichuris suis: a secretory chymotrypsin/elastase inhibitor with potential as an immunomodulator. Exp. Parasitol. $95,36-44$

24 Whisstock, J.et al. (1998) An atlas of serpin conformations. Trends Biochem. Sci. 23, 63-67

25 Huntington, J.A. et al. (2000) Structure of a serpin-protease complex shows inhibition by deformation. Nature 407, 923-926

26 Hopkins, P.C.R. and Whisstock, J. (1994) Function of maspin. Science 265, 1893-1894

27 Goodwin, R.L.et al. (1996) Patterns of divergence during evolution of $\alpha 1$-proteinase inhibitors in mammals. Mol. Biol. Evol. 13, 346-358

28 Hill, R.E. and Hastie, N.D. (1987) Accelerated evolution in the reactive centre regions of serine protease inhibitors. Nature 326, 96-99

29 Strandberg, L. et al. (1988) The organization of the human-plasminogen-activator-inhibitor-1 gene: implications on the evolution of the serine-protease inhibitor family. Eur. J. Biochem. 176, 609-616
30 Gilbert, W. et al. (1997) Origin of genes. Proc. Natl. Acad. Sci. U. S. A. 94, 7698-7703

31 Stoltzfus, A. et al. (1994) Testing the exon theory of genes: the evidence from protein structure. Science 265, 202-207

32 Duggan, B.M. et al. (1999) Inherent flexibility in a potent inhibitor of blood coagulation, recombinant nematode anticoagulant protein c2. Eur. J. Biochem. 265, 539-548

33 Hawley, J.H. and Peanasky, R.J. (1992) Ascaris suum: are trypsin inhibitors involved in species specificity of Ascarid nematodes? Exp. Parasitol. $75,112-118$

34 Zang, X. et al. (2000) The serpin secreted by Brugia malayi microfilariae, Bm-SPN-2, elicits strong, but short-lived, immune responses in mice and humans. J. Immunol. 165, 5161-5169

35 Chertov, O. et al. (1997) Identification of human neutrophil-derived cathepsin $\mathrm{G}$ and azurocidin/CAP37 as chemoattractants for mononuclear cells and neutrophils. J. Exp. Med. 186, 739-747

36 Padrines, M. et al. (1994) Interleukin-8 processing by neutrophil elastase, cathepsin $\mathrm{G}$ and proteinase-3. FEBS Lett. 352, 231-235

37 Modha, J.et al. (1996) Schistosomes and serpins: a complex business. Parasitol. Today 12, 119-121

38 Martzen, M.R. et al. (1985) Ascaris suum: localization by immunochemical and fluorescent probes of host proteases and parasite proteinase inhibitors in cross-sections. Exp. Parasitol. 60, 139-149

39 Cappello, M.et al. (1995) Ancylostoma caninum anticoagulant peptide: a hookworm-derived inhibitor of human coagulation factor Xa. Proc. Natl. Acad. Sci. U. S. A. 92, 6152-6156

40 Rebello, S.S. et al. (1997) Antithrombotic efficacy of a recombinant nematode anticoagulant peptide (rNAP5) in canine models of thrombosis after single subcutaneous administration. J. Pharmacol. Exp. Ther. 283, 91-99

41 Jeannin, P. et al. (1998) $\alpha 1$ antitrypsin upregulates human B cell differentiation selectively into IgE- and IgG4-secreting cells. Eur. J. Immunol. 28, 1815-1822

$42 \mathrm{Li}$, Z. et al. (1995) Preferential recognition by human IgE and IgG4 of a species-specific Schistosoma haematobium serine protease inhibitor. J. Infect. Dis. 171, 416-422 